\title{
ON AN EXACT WKB APPROACH TO ABLOWITZ-SEGUR'S CONNECTION PROBLEM FOR THE SECOND PAINLEVÉ EQUATION
}

\author{
YOSHITSUGU TAKEI ${ }^{1}$
}

(Received 24 May, 2000; revised 7 September, 2000)

\begin{abstract}
We discuss Ablowitz-Segur's connection problem for the second Painlevé equation from the viewpoint of $\mathrm{WKB}$ analysis of Painlevé transcendents with a large parameter. The formula they first discovered is rederived from a suitable combination of connection formulas for the first Painlevé equation.
\end{abstract}

\section{Introduction}

Exact WKB analysis, initiated by Voros ([19]) and developed by Pham and his collaborators $([5,9]$ etc. $)$, is a powerful tool for analyzing the global behaviour of solutions of one-dimensional Schrödinger equations (see [13] also). In parallel to the case of Schrödinger equations, Aoki, Kawai and the author have recently established an analogous analysis for Painlevé transcendents (that is, solutions of Painlevé equations) with a large parameter $([3,12,14,17])$. Although mathematically rigorous justification of the theory is still an open problem, this analysis has gradually turned out to be effective for studying global connection problems in Painlevé equations. In this paper, to show both validity and effectiveness, we explain an outline of the theory and discuss Ablowitz-Segur's connection problem for the second Painlevé equation from this viewpoint.

\section{Review of WKB analysis of Painlevé transcendents}

The equations treated in our WKB analysis of Painleve transcendents are the following Painlevé equations $\left(P_{J}\right)$ with a large parameter $\eta$ :

$$
d^{2} \lambda / d t^{2}=\eta^{2} F_{J}(\lambda, t)+G_{J}(\lambda, d \lambda / d t, t) \quad(J=\mathrm{I}, \ldots, \mathrm{VI}),
$$

\footnotetext{
'Research Institute for Mathematical Sciences, Kyoto University, Kyoto, 606-8502, Japan; e-mail: takei@kurims.kyoto-u.ac.jp.
}

(C) Australian Mathematical Society 2002, Serial-fee code 1446-1811/02 
where $F_{J}$ and $G_{J}$ are rational functions. For example, $\left(P_{1}\right): d^{2} \lambda / d t^{2}=\eta^{2}\left(6 \lambda^{2}+t\right)$, $\left(P_{\mathrm{II}}\right): d^{2} \lambda / d t^{2}=\eta^{2}\left(2 \lambda^{3}+t \lambda+c\right)$.

For these equations $\left(P_{J}\right)$ we can construct the following. 2-parameter family of formal solutions called instanton-type solutions $([3,16])$ :

$$
\lambda_{J}(t ; \alpha, \beta)=\lambda_{0}(t)+\eta^{-1 / 2} \lambda_{1 / 2}(t, \eta)+\eta^{-1} \lambda_{1}(t, \eta)+\cdots,
$$

where $\lambda_{0}(t)$ is an algebraic function determined by $F_{J}\left(\lambda_{0}(t), t\right)=0$ and $\lambda_{j / 2}(t, \eta)$ $(j \geq 1)$ has the expansion

$$
\begin{aligned}
& \lambda_{1 / 2}=\mu_{J}(t)\left\{\alpha\left(\theta_{J}(t) \eta^{2}\right)^{\alpha \beta} e^{\eta \phi_{J}(t)}+\beta\left(\theta_{J}(t) \eta^{2}\right)^{-\alpha \beta} e^{-\eta \phi_{J}(t)}\right\}, \\
& \lambda_{j / 2}=\sum_{k=0}^{j} b_{j-2 k}^{(j / 2)}(t)\left(\theta_{J}(t) \eta^{2}\right)^{(j-2 k) \alpha \beta} e^{(j-2 k) \eta \phi_{J}(t)} \quad(j \geq 2) .
\end{aligned}
$$

Here

$$
\phi_{J}(t)=\int_{t_{0}}^{t} \sqrt{\frac{\partial F_{J}}{\partial \lambda}\left(\lambda_{0}(t), t\right)} d t \quad\left(t_{0}: \text { a fixed point }\right)
$$

$\mu_{J}(t)$ and $\theta_{J}(t)$ are some functions of $t$ (whose explicit description is given in [14]), and $(\alpha, \beta)$ denotes a pair of free parameters. (Precisely speaking, both $\alpha$ and $\beta$ are infinite series $\sum_{n \geq 0} \eta^{-n} \alpha_{n}$ and $\sum_{n \geq 0} \eta^{-n} \beta_{n}$ since $b_{ \pm 1}^{(n+1 / 2)}(t)(n \geq 1)$ may contain additional free parameters $\left(\alpha_{n}, \beta_{n}\right)$. In this paper, however, we are concerned only with computations of the top degree level and accordingly consider $\alpha$ and $\beta$ as ordinary parameters.)

Instanton-type solutions can be regarded as a substitute for WKB solutions of Schrödinger equations. For these solutions let us introduce the notion of turning points and Stokes curves in the following way.

DEFINITION 1. (i) A turning point of $\left(P_{J}\right)$ is a point $r$ satisfying

$$
F_{J}\left(\lambda_{0}(r), r\right)=\frac{\partial F_{J}}{\partial \lambda}\left(\lambda_{0}(r), r\right)=0 .
$$

A turning point $r$ is said to be simple if $\left(\partial^{2} F_{J} / \partial \lambda^{2}\right)\left(\lambda_{0}(r), r\right) \neq 0$.

(ii) A Stokes curve of $\left(P_{J}\right)$ is defined by the relation

$$
\Im \int_{r}^{t} \sqrt{\frac{\partial F_{J}}{\partial \lambda}\left(\lambda_{0}(t), t\right)} d t=0,
$$

where $r$ is a turning point of $\left(P_{J}\right)$.

For example, for $\left(P_{\mathrm{I}}\right) t=0$ is a unique simple turning point and the Stokes curves are given by the relation $\Im x^{5 / 4}=0$ (see Figure 1 below). 


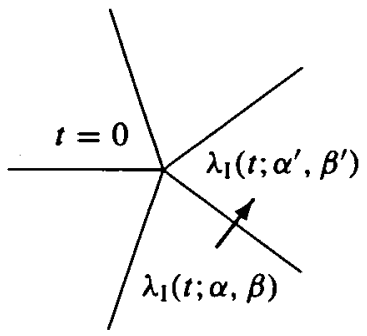

FIGURE 1. Here $\lambda_{\mathrm{I}}(t ; \alpha, \beta)$ is an instanton-type solution of $\left(P_{1}\right)$ in the region $\{-3 \pi / 5<\arg t<-\pi / 5)$; $\lambda_{1}\left(t ; \alpha^{\prime}, \beta^{\prime}\right)$ is its analytic continuation to $\{-\pi / 5<\arg t<\pi / 5\}$.

Stokes curves divide the complex $t$-plane (or rather the Riemann surface of $F_{J}(\lambda, t)=0$ ) into several small regions called Stokes regions. In the case of Schrödinger equations, by employing the Borel resummation technique, we find that a (Borel resummed) WKB solution represents an analytic solution in each Stokes region. Similarly an instanton-type solution $\lambda_{J}(t ; \alpha, \beta)$ of $\left(P_{J}\right)$ should be expected to represent a true solution in a Stokes region, although it is not known how to give it an analytic meaning at the present stage. The so-called "connection formula", that is, a formula describing the relation between the instanton-type solutions in two adjacent regions corresponding to the same analytic solution, then plays a crucially important role in analyzing the global behaviour of solutions of $\left(P_{J}\right)$. The following theorem may reduce the determination of the explicit form of the connection formula for $\left(P_{J}\right)$ to that for $\left(P_{\mathrm{I}}\right)$.

THEOREM 2.1 (Local reduction to $\left(P_{1}\right) ;[14]$ ). In a neighbourhood of $\tilde{t}_{*}$, a point on a Stokes curve emanating from a simple turning point $\tilde{r}$ of $\left(P_{J}\right)$, the Painlevé equation $\left(P_{J}\right)$ can be formally transformed to $\left(P_{\mathrm{I}}\right)$. To be more precise, for each instanton-type solution $\tilde{\lambda}_{J}(\tilde{i} ; \tilde{\alpha}, \tilde{\beta})$ of $\left(P_{J}\right)$ we can find an instanton-type solution $\lambda_{\mathrm{I}}(t ; \alpha, \beta)$ of $\left(P_{1}\right)$ for which the following holds:

$$
x(\tilde{\lambda} J(\tilde{t} ; \tilde{\alpha}, \tilde{\beta}), \tilde{t}, \eta)=\lambda_{1}(t(\tilde{t}, \eta) ; \alpha, \beta),
$$

where $x(\tilde{x}, \tilde{t}, \eta)$ and $t(\tilde{t}, \eta)$ are formal series of the form

$$
x(\tilde{x}, \tilde{t}, \eta)=\sum_{j \geq 0} \eta^{-j / 2} x_{j / 2}(\tilde{x}, \tilde{t}, \eta), \quad t(\tilde{t}, \eta)=\sum_{j \geq 0} \eta^{-j / 2} t_{j / 2}(\tilde{t}, \eta),
$$

and the parameters obey the relation

$$
(\alpha, \beta)=\left(i^{\kappa} \tilde{\alpha}, i^{\kappa} \tilde{\beta}\right)+\mathscr{O}\left(\eta^{-1}\right)
$$

with some integer $\kappa$. (Here we are assuming that the end-point $t_{0}$ in the definition (2) of $\tilde{\phi}_{J}(\tilde{t})$ and $\phi_{\mathrm{I}}(t)$ is taken to be $\tilde{r}$ and 0 respectively.) 
For the canonical equation $\left(P_{\mathrm{l}}\right)$ we have the following connection formula. (Here we discuss only a Stokes curve $\{\arg t=-\pi / 5\}$ as there is no essential difference between the five Stokes curves due to symmetry.)

THEOREM 2.2 (Connection formula for $\left.\left(P_{1}\right) ;[17]\right)$. Let $\lambda_{I}(t ; \alpha, \beta)$ (normalized so that $t_{0}=0$ in (2)) be an instanton-type solution of $\left(P_{1}\right)$ in the region $\{-3 \pi / 5<$ $\arg t<-\pi / 5\}$ and $\lambda_{\mathrm{I}}\left(t ; \alpha^{\prime}, \beta^{\prime}\right)$ its analytic continuation to $\{-\pi / 5<\arg t<\pi / 5\}$ (see Figure 1). Then the following relations hold between $(\alpha, \beta)$ and $\left(\alpha^{\prime}, \beta^{\prime}\right)$ :

$$
\left\{\begin{array}{l}
\alpha e^{-i \pi E / 4} \chi(E)=\alpha^{\prime} e^{-i \pi E^{\prime} / 4} \chi\left(E^{\prime}\right), \\
e^{i \pi E / 2}+\beta e^{i \pi E / 2} \chi(-E)=\beta^{\prime} e^{i \pi E^{\prime} / 2} \chi\left(-E^{\prime}\right),
\end{array}\right.
$$

where $\chi(z)=\sqrt{\pi} 2^{z / 4+1} / \Gamma(z / 4+1), E=-8 \alpha \beta$ and $E^{\prime}=-8 \alpha^{\prime} \beta^{\prime}$.

For a given instanton-type solution $\lambda_{\mathrm{I}}(t ; \alpha, \beta)$ in $\{-3 \pi / 5<\arg t<-\pi / 5\}$ its analytic continuation across $\{\arg t=-\pi / 5\}$ should be obtained by solving the relation (8) with respect to $\left(\alpha^{\prime}, \beta^{\prime}\right)$. Note that (8) is not uniquely solvable for $\left(\alpha^{\prime}, \beta^{\prime}\right)$. This kind of non-uniqueness is related to analytic interpretation of instanton-type solutions (see [18]). Establishing the analytic version of these theorems is still an open problem.

In parallel with the Schrödinger case, the connection formula at a simple turning point for $\left(P_{J}\right)$ should, in principle, be derived from a combination of these two theorems (that is, the formula for $\left(P_{J}\right)$ should be obtained by substitution of (7) into (8)). Repeated use of the formula thus obtained according to the configuration of Stokes curves should then enable us to solve global connection problems for $\left(P_{J}\right)$. In the subsequent sections, to exemplify the validity of our approach, we discuss Ablowitz-Segur's connection problem from this viewpoint.

\section{Ablowitz-Segur's connection problem}

Let us consider a solution $u(z)$ of the equation

$$
u^{\prime \prime}=z u+2 u^{3}
$$

with the following asymptotic behaviour for $z>0, z \rightarrow \infty$ :

$$
u(z) \sim \frac{a}{2 \sqrt{\pi}} z^{-1 / 4} e^{-2 z^{3 / 2} / 3} \quad(z \rightarrow+\infty)
$$

where $a$ is a constant satisfying $0<a<1$. It is known that, after the analytic continuation along the real axis, $u(z)$ has the following asymptotic expansion for $z \rightarrow-\infty$ :

$$
u(z) \sim d(-z)^{-1 / 4} \sin \left(\frac{2}{3}(-z)^{3 / 2}-\frac{3}{4} d^{2} \log (-z)+\theta\right) \quad(z \rightarrow-\infty),
$$


where $d$ and $\theta$ are given by

$$
\left\{\begin{array}{l}
d^{2}=-\frac{1}{\pi} \log \left(1-a^{2}\right) \\
\theta=\frac{\pi}{4}-\frac{3}{2} d^{2} \log 2-\arg \Gamma\left(1-\frac{i d^{2}}{2}\right) .
\end{array}\right.
$$

The formula (12) was first discovered by Ablowitz and Segur ([1, 15]), then discussed by many people (for example, [6] and especially [11] are closely related to our approach in the sense that they discuss a singular-perturbative form of (9)), and finally proved rigorously in [7] and [8]. See also, for example, [4] for more recent research.

Our goal is to derive this formula (12) by using the WKB analysis explained in the preceding section.

\section{WKB analysis for the second Painlevé equation}

By a simple scaling transformation

$$
u=\eta^{1 / 3} \lambda, \quad z=\eta^{2 / 3} t
$$

(9) is transformed to our second Painlevé equation

$$
\frac{d^{2} \lambda}{d t^{2}}=\eta^{2}\left(2 \lambda^{3}+t \lambda\right)
$$

with the parameter $c$ being equal to 0 . Furthermore, taking a solution of $\left(P_{\mathrm{II}}^{0}\right)$ of instanton type $\lambda_{\mathrm{II}}^{0}(t ; \alpha, \beta)$ with identically vanishing top term $\lambda_{0}(t) \equiv 0$ and $\phi_{\mathrm{II}}^{0}(t)=$ $\int_{0}^{t} \sqrt{t} d t$, we readily find that both the asymptotic solutions (10) and (11) of (9) correspond through the scaling transformation (13) to $\lambda_{\mathrm{II}}^{0}(t ; \alpha, \beta)$ (at least at the leading order level) on the condition that the parameters are related in the following manner:

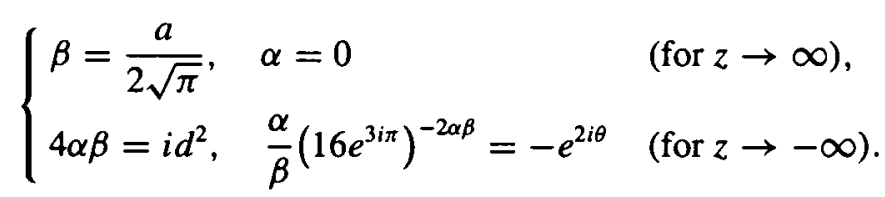

Our problem is thus to solve the connection problem between $\lambda_{\mathrm{II}}^{0}(t ; 0, \beta)$ for $z \rightarrow \infty$ and $\lambda_{\text {II }}^{0}(t ; \alpha, \beta)$ for $z \rightarrow-\infty$.

Let us here draw the configuration of Stokes curves of the second Painleve equation ( $P_{\mathrm{II}}$ ) (see Figure 2). In the case of $c=0$ there is a unique turning point at $t=0$ and three Stokes curves (lines) emanate from it. The connection problem in question can be solved using connection formulas on these Stokes curves. However, it is not an 

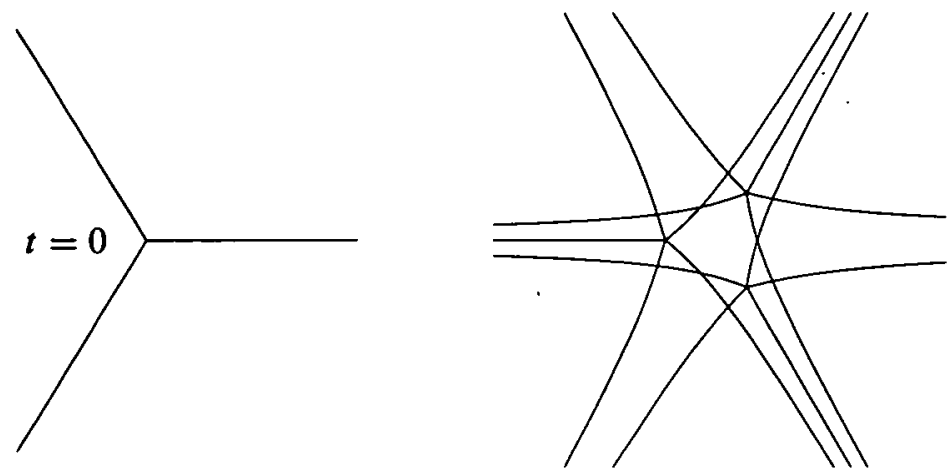

FIGURE 2. Stokes curves of ( $\left.P_{\mathrm{II}}\right)$ for $c=0$ (left) and for $c \neq 0$ (right).

easy task to determine their explicit form as $t=0$ is not a simple turning point. On the other hand, in the case of $c \neq 0$ (although the configuration itself is quite complicated) all of the three turning points $r_{j}=-6(c / 4)^{2 / 3} \omega^{j}\left(j=0,1,2, \omega=e^{2 i \pi / 3}\right)$ are simple and hence the connection formula $(8)$ for $\left(P_{\mathrm{I}}\right)$ is expected to be applicable to each Stokes curve. Taking this situation into account, we first consider $\left(P_{\mathrm{II}}\right)$ with a non-zero parameter $c$ and then take a limit $c \rightarrow 0$ to study the connection problem for $\left(P_{\mathrm{II}}^{0}\right)$.

As is pointed out and explicitly done using Mathematica 3.0.1 in [2], the configuration of Stokes curves of $\left(P_{\mathrm{II}}\right)$ should be lifted onto the Riemann surface of $F_{\mathrm{II}}(\lambda, t)=2 \lambda^{3}+t \lambda+c=0$. (See Figure 3 , where a wiggly line, a solid line and a dotted line respectively designate a cut, a Stokes curve on the sheet concerned and that on another sheet.) Let us consider a limit $c \rightarrow 0$ for this lift of Stokes curves. In the process of $c \rightarrow 0$ all of the turning points tend to the origin and the cut between the second and third sheets disappears. Consequently the Riemann surface at $c=0$ becomes the disjoint union of two connected components; the double-covering part (first and second sheets) and the simple-covering part (third sheet). Note that this is consistent with the fact that $F_{\mathrm{II}}(\lambda, t)$ at $c=0$ may be factorized as $2 \lambda^{3}+t \lambda=\lambda\left(2 \lambda^{2}+t\right)$. Since we are interested in instanton-type solutions of $\left(P_{\mathrm{II}}^{0}\right)$ with identically vanishing top term $\lambda_{0}(t) \equiv 0$, we should discuss instanton-type solutions of $\left(P_{11}\right)$ on the third sheet for $c \neq 0$. The configuration of Stokes curves on the third sheet, in fact, approaches that of $\left(P_{\mathrm{II}}^{0}\right)$.

Having these geometric facts in mind, we now try to determine the connection formula for $\left(P_{\mathrm{II}}^{0}\right)$ on, for example, the positive real axis, that is, the relation between $\lambda_{\mathrm{II}}^{0}(t ; \alpha, \beta)$ in the region $\{-2 \pi / 3<\arg t<0\}$ and $\lambda_{\mathrm{II}}^{0}\left(t ; \alpha^{\prime}, \beta^{\prime}\right)$ in $\{0<\arg t<2 \pi / 3\}$. Let $\lambda_{\mathrm{II}}(t ; \alpha, \beta)$ and $\lambda_{\mathrm{II}}\left(t ; \alpha^{\prime}, \beta^{\prime}\right)$ respectively denote instanton-type solutions of $\left(P_{\mathrm{II}}\right)$ in the corresponding regions on the third sheet. (See Figure 3 . Here we assume that the end-point $t_{0}$ in (2) is taken to be a simple turning point $r_{2}$ for both the solutions.) 

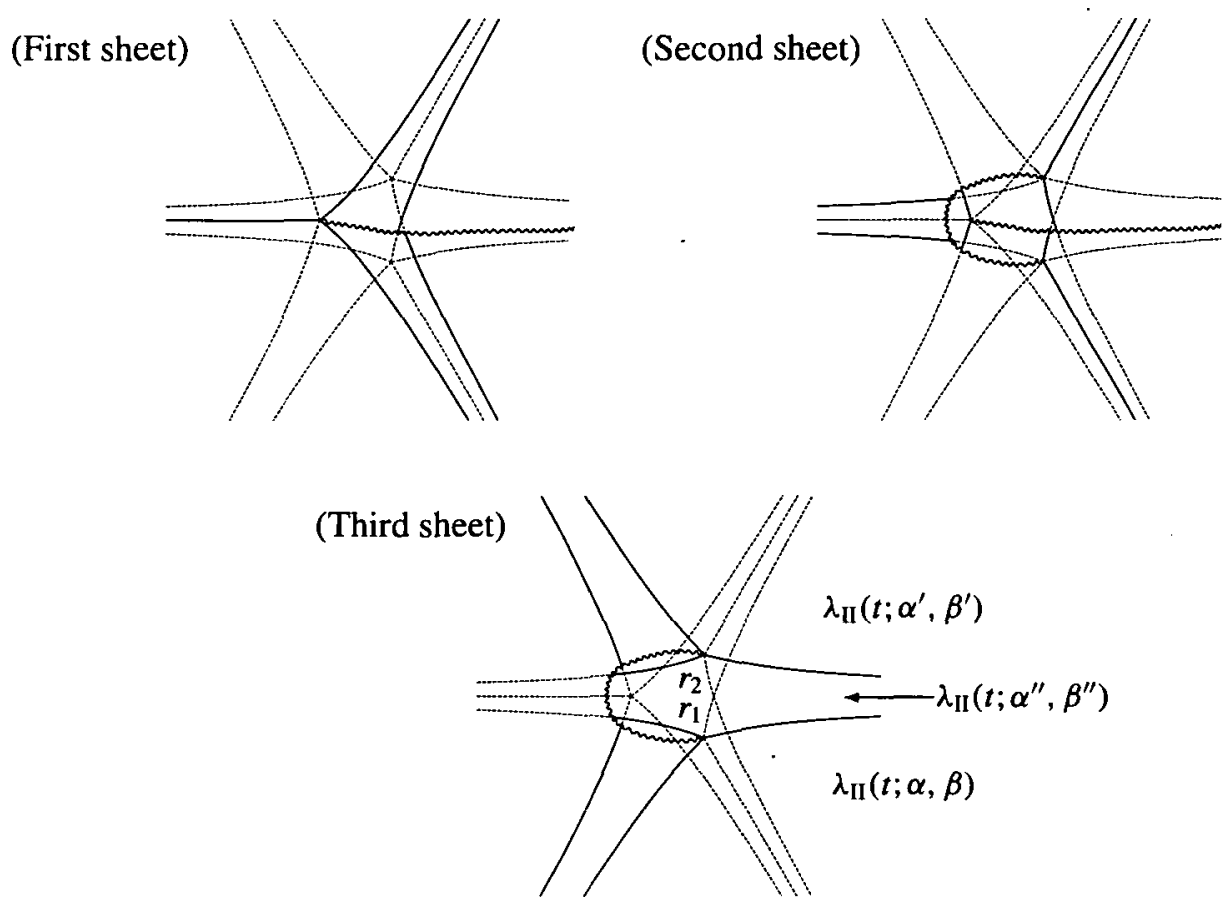

FIGURE 3. The lift of Stokes curves of $\left(P_{\mathrm{II}}\right)$ onto the Riemann surface of $2 \lambda^{3}+t \lambda+c=0$.

Note that there is an intermediate region between these two regions, an instanton-type solution in which is denoted by $\lambda_{\mathrm{II}}\left(t ; \alpha^{\prime \prime}, \beta^{\prime \prime}\right)$. It then follows from the simplicity of the turning point $r_{2}$ that the relation between $\lambda_{\mathrm{II}}\left(t ; \alpha^{\prime \prime}, \beta^{\prime \prime}\right)$ and $\lambda_{\mathrm{II}}\left(t ; \alpha^{\prime}, \beta^{\prime}\right)$ should be described by the connection formula $(8)$ for $\left(P_{1}\right)$, provided that our expectation explained at the end of Section 2 is true, that is,

$$
\left\{\begin{array}{l}
\alpha^{\prime \prime} e^{-i \pi E^{\prime \prime} / 4} \chi\left(E^{\prime \prime}\right)=\alpha^{\prime} e^{-i \pi E^{\prime} / 4} \chi\left(E^{\prime}\right), \\
e^{i \pi E^{\prime \prime} / 2}+\beta^{\prime \prime} e^{i \pi E^{\prime \prime} / 2} \chi\left(-E^{\prime \prime}\right)=\beta^{\prime} e^{i \pi E^{\prime} / 2} \chi\left(-E^{\prime}\right) .
\end{array}\right.
$$

On the other hand, considering the local reduction to $\left(P_{\mathrm{I}}\right)$ at $r_{1}$, since the integer $\kappa$ in the relation (7) of parameters is equal to 2 and the end-point $t_{0}$ of $\phi_{\mathrm{II}}(t)$ is now chosen to be $r_{2}$, the parameters obey the relation (at the top degree level with respect to $\eta$ )

$$
(\alpha, \beta)=\left(-\tilde{\alpha} \exp \left(\eta \int_{r_{2}}^{r_{1}} \sqrt{6 \lambda_{0}^{2}+t} d t\right),-\tilde{\beta} \exp \left(-\eta \int_{r_{2}}^{r_{1}} \sqrt{6 \lambda_{0}^{2}+t} d t\right)\right) .
$$

Hence, as $\int_{r_{2}}^{r_{1}} \sqrt{6 \lambda_{0}^{2}+t} d t=2 i \pi c$ holds, the relation between $\lambda_{\mathrm{II}}(t ; \alpha, \beta)$ and 
$\lambda_{\text {II }}\left(t ; \alpha^{\prime \prime}, \beta^{\prime \prime}\right)$ should be described by

$$
\left\{\begin{array}{l}
\alpha e^{-i \pi E / 4} \chi(E)=\alpha^{\prime \prime} e^{-i \pi E^{\prime \prime} / 4} \chi\left(E^{\prime \prime}\right) \\
-e^{i \pi E / 2} e^{2 i \pi c \eta}+\beta e^{i \pi E / 2} \chi(-E)=\beta^{\prime \prime} e^{i \pi E^{\prime \prime} / 2} \chi\left(-E^{\prime \prime}\right)
\end{array}\right.
$$

Eliminating ( $\left.\alpha^{\prime \prime}, \beta^{\prime \prime}\right)$ from (15) and (17) and taking a limit $c \rightarrow 0$, we thus obtain the "connection formula for $\left(P_{\mathrm{II}}^{0}\right)$ on the positive real axis"

$$
\left\{\begin{array}{l}
\alpha e^{-i \pi E / 4} \chi(E)=\alpha^{\prime} e^{-i \pi E^{\prime} / 4} \chi\left(E^{\prime}\right), \\
e^{i \pi E / 2}-\beta e^{i \pi E / 2} \chi(-E)=\frac{1-\beta^{\prime} e^{i \pi E^{\prime} / 2} \chi\left(-E^{\prime}\right)}{1-i \alpha^{\prime} e^{-i \pi E^{\prime} / 4} \chi\left(E^{\prime}\right)}
\end{array}\right.
$$

(Note that $\alpha=\alpha^{\prime}=0$ and $\beta=\beta^{\prime}$ satisfy (18). This reflects the well-definedness of $\lambda_{\text {II }}^{0}(t ; 0, \beta)$ on the positive real axis.)

Connection formulas on the other Stokes curves can also be derived from (18) using the rotational symmetry of angle $2 \pi / 3$ of $\left(P_{\mathrm{II}}^{0}\right)$. For example, replacements $\alpha \mapsto-i \beta$, $\beta \mapsto-i \alpha$ in (18) give the following connection formula on $\arg t=2 \pi / 3\}$ :

$$
\left\{\begin{array}{l}
\beta e^{i \pi E / 4} \chi(-E)=\beta^{\prime} e^{i \pi E^{\prime} / 4} \chi\left(-E^{\prime}\right) \\
e^{-i \pi E / 2}+i \alpha e^{-i \pi E / 2} \chi(E)=\frac{1+i \alpha^{\prime} e^{-i \pi E^{\prime} / 2} \chi\left(E^{\prime}\right)}{1-\beta^{\prime} e^{i \pi E^{\prime} / 4} \chi\left(-E^{\prime}\right)}
\end{array}\right.
$$

Putting $\alpha=0$ in (19), we finally obtain the following solution of the connection problem in question: Consider an instanton-type solution $\lambda_{\mathrm{II}}^{0}(t ; 0, \beta)$ of $\left(P_{\mathrm{II}}^{0}\right)$ on the positive real axis (which is well-defined as mentioned above) and denote by $\lambda_{\text {II }}^{0}\left(t ; \alpha^{\prime}, \beta^{\prime}\right)$ its analytic continuation to the negative real axis through the upper-half plane. Then we have

$$
\left\{\begin{array}{l}
2 \sqrt{\pi} \beta=-i \alpha^{\prime} e^{-i \pi E^{\prime} / 2} \chi\left(E^{\prime}\right) \\
2 \sqrt{\pi} \beta=\beta^{\prime} e^{i \pi E^{\prime} / 4} \cdot \chi\left(-E^{\prime}\right)
\end{array}\right.
$$

By a straightforward computation we find that the formula (12) certainly follows from (20) and (14) (assuming that $(\alpha, \beta)$ in the second relation of (14) is replaced by $\left(\alpha^{\prime}, \beta^{\prime}\right)$ ). In other words, Ablowitz-Segur's formula can be derived from repeated use of the connection formula for $\left(P_{\mathrm{l}}\right)$.

We believe that this result strongly supports the validity and effectiveness of our WKB theory for Painlevé equations. To complete the theory, however, we need an analytic interpretation of instanton-type solutions. (A recent work of Joshi ([10]) may be regarded as the first step in the study of this problem.) Clarifying the precise analytic meaning of the local reduction to $\left(P_{\mathrm{I}}\right)$ is also an important open problem in this theory. 
Acknowledgement The author would like to express his sincere gratitude to Professors T. Kawai and T. Aoki for the stimulating discussions he enjoyed with them. This work is supported in part by the Japan Society for the Promotion of Science under Grant-in-Aid for Encouragement of Young Scientists (No. 11740087).

\section{References}

[1] M. J. Ablowitz and H. Segur, "Asymptotic solution of the Korteweg-de Vries equation", Stud. Appl. Math. 57 (1977) 13-44.

[2] T. Aoki, "Stokes geometry of Painlevé equations with a large parameter", RIMS Kôkyûroku 1088 (1999) 39-54.

[3] T. Aoki, T. Kawai and Y. Takei, "WKB analysis of Painlevé transcendents with a large parameter. II", in Structure of Solutions of Differential Equations (eds. M. Morimoto and T. Kawai), (World Scientific, 1996) 1-49.

[4] A. P. Bassom, P. A. Clarkson, C. K. Law and J. B. McLeod, "Application of uniform asymptotics to the second Painlevé transcendent", Arch. Rat. Mech. Anal. 143 (1998) 241-271.

[5] B. Candelpergher, J. C. Nosmas and F. Pham, Approche de la résurgence (Hermann, 1993).

[6] P. A. Clarkson and J. B. McLeod, "A connection formula for the second Painlevé transcendent", Arch. Rat. Mech. Anal. 103 (1988) 97-138.

[7] P. Deift and X. Zhou, "A steepest descent method for oscillatory Riemann-Hilbert problems", Ann. Math. 137 (1993) 295-368.

[8] P. Deift and X. Zhou, “Asymptotics for the Painlevé II equation”, Comm. Pure Appl. Math. 48 (1995) 277-337.

[9] E. Delabaere and F. Pham, "Resurgent methods in semi-classical asymptotics", Ann. Inst. H. Poincaré 71 (1999) 1-94.

[10] N. Joshi, "True solutions asymptotic to formal WKB solutions of the second Painlevé equation with large parameter", in Toward the Exact WKB Analysis of Differential Equations, Linear or Non-Linear (eds. C. J. Howls, T. Kawai and Y. Takei), (Kyoto Univ. Press, 2000) 223-230.

[11] M. V. Karasev and A. V. Pereskokov, "On connection formulas for the second Painlevé transcendent”, Russian Acad. Sci. Izv. Math. 42 (1994) 501-560.

[12] T. Kawai and Y. Takei, "WKB analysis of Painlevé transcendents with a large parameter. I", Adv. in Math. 118 (1996) 1-33.

[13] T. Kawai and Y. Takei, Algebraic Analysis of Singular Perturbations (Iwanami, 1998), (In Japanese. English translation will be published by Amer. Math. Soc.).

[14] T. Kawai and Y. Takei, "WKB analysis of Painlevé transcendents with a large parameter. III", Adv. in Math. 134 (1998) 178-218.

[15] H. Segur and M. J. Ablowitz, "Asymptotic solutions of nonlinear evolution equations and a Painlevé transcendent", Physica D 3 (1981) 165-184.

[16] Y. Takei, "Singular-perturbative reduction to Birkhoff normal form and instanton-type formal solutions of Hamiltonian systems", Publ. RIMS, Kyoto Univ. 34 (1998) 601-627.

[17] Y. Takei, "An explicit description of the connection formula for the first Painlevé equation", in Toward the Exact WKB Analysis of Differential Equations, Linear or Non-Linear (eds. C. J. Howls, T. Kawai and Y. Takei), (Kyoto Univ. Press, 2000) 271-296.

[18] Y. Takei, "The space of initial conditions of Painlevé equations and WKB analysis", RIMS Kôkyûroku 1133 (2000) 104-116, (In Japanese).

[19] A. Voros, "The return of the quartic oscillator. The complex WKB method", Ann. Inst. H. Poincaré 39 (1983) 211-338. 